\title{
Targeting Cancer Stem Cells with Nanoparticle-Enabled Therapies
}

\author{
Andrew R. Burke ${ }^{1}$, Ravi N. Singh ${ }^{2}$, David L. Carroll ${ }^{3}$, Frank M. Torti ${ }^{4}$ and Suzy V. Torti ${ }^{5 *}$
}

${ }^{1}$ Tumor Angiogenesis Section, Mouse Cancer Genetics Program, Frederick National Laboratory for Cancer Research, National Institutes of Health, Frederick, MD 21702, USA ${ }^{2}$ Department of Cancer Biology, Wake Forest School of Medicine, Winston Salem, NC 27157, USA

${ }^{3}$ Department of Physics, Wake Forest University, Winston Salem, NC 27109, USA

${ }^{4}$ Department of Medicine, University of Connecticut School of Medicine, Farmington, CT, 06030, USA

${ }^{5}$ Department of Molecular, Microbial and Structural Biology, University of Connecticut School of Medicine, Farmington, CT, 06030, USA

\begin{abstract}
Emerging evidence suggests that multiple tumor types are sustained by a small population of transformed stemlike cells that have the ability to both self-renew and give rise to non-tumorigenic daughter cells that constitute the bulk of a tumor. These cells, which generally constitute a minority of the overall cancer cell population, are highly resistant to conventional therapies and persist following treatment, leading to disease relapse and the formation of distant metastases. Therapies that disrupt the maintenance and survival of cancer stem cells are the subject of active current investigation. This review discusses recent approaches to the application of nanomedicine to the targeting and elimination of cancer stem cells. Specifically, recent publications in the areas of nanoparticle-enabled drug and nucleic acid delivery and photothermal therapy are addressed.
\end{abstract}

Keywords: Cancer stem cells; Tumor-initiating cells; Drug delivery; Nucleic acid delivery; Targeted; Nanoparticle; Carbon nanotube; Photo thermal therapy; Hyperthermia

\section{Introduction}

The intratumoral heterogeneity of cancer cells presents a major challenge to the development of effective cancer therapies. However, a growing body of evidence suggests that tumors may be driven by a small population of transformed stem-like cells with the ability to undergo both self-renewal and differentiation into the diverse cancer cell population that constitutes the bulk of the tumor [1-4]. In 1997, Bonnet and Dick identified a single cell isolated from a bulk cancer cell population capable of initiating cancers that recapitulated the cellular heterogeneity of the parent pathology when transferred into an immune compromised animal model [5]. In their work, the authors demonstrated that acute myelogenous leukemias (AML) could be initiated in NOD/SCID mice through the transplantation of a rare $\left(<0.2 \%\right.$ of whole cell population) $\mathrm{CD} 34^{+} / \mathrm{CD} 38^{-}$cell from human donors. Only cells displaying these markers were capable of engrafting and generating the cellular diversity evident in human AML. In 2003, Al-Hajj et al. demonstrated this cellular hierarchy extended to solid tumors by showing that a diverse set of human breast cancer specimens could be fractionated by surface markers and that only cells displaying the $\mathrm{CD} 44^{\text {hi }} / \mathrm{CD} 24^{\text {low }} /$ Lin antigen profile could form tumors in immune compromised mice [6]. Moreover, these cells displayed self-renewal and multi-lineage differentiation abilities in long-term in vitro cultures [6]. Since these seminal publications, cells displaying similar capabilities have been isolated from a range of human tumors including: brain, colon, head and neck, lung, melanoma, pancreatic, prostate and kidney [3,7-14].

Current research suggests these so-called cancer stem cells (CSCs) or tumor initiating cells (TICs) survive standard chemo and radiotherapies and persist following treatment $[15,16]$. As these cells are both invasive and highly tumorigenic it has been hypothesized that the inability to efficiently eliminate CSCs during conventional therapy may result in disease relapse and formation of metastases. New treatment modalities in the form of molecularly-directed nanomedicines (purpose-built constructs having principal dimensions of 1-100 nm) with the potential to deliver therapeutic payloads directly to CSCs are currently being described in the primary research literature
$[17,18]$. Table 1 (Included as supplementary data) summarizes many of these nanomaterials and discusses their significance in greater detail. With several nanomedicines entering early stage clinical trials, it is anticipated that their ability to selectively target and kill the cellular drivers of tumor progression will fundamentally alter the clinical management of cancer. Accordingly, this review highlights recent advances in the area of nanomedicine with a specific focus on nanoparticle-mediated therapeutic delivery to CSCs and the response of those cells to such treatments.

\section{Review of Literature}

\section{Nanoparticle platforms for CSC-targeted drug delivery}

Nanoscale drug delivery technologies offer fundamental advantages over contemporary small molecule pharmaceuticals used in clinical practice. These advantages include increased bioavailibity, extended drug half-life and reduced off-target toxicities [17]. Furthermore, the new generation of therapeutic nanoparticles is inherently multifunctional: combining active drug compounds with selective targeting moieties and, in many cases, imaging agents that permit localization by standard $\mathrm{x}$-ray, magnetic resonance (MR) or positron emission tomography (PET) technologies. These so called "theranostic" constructs offer the promise of diminished drug toxicity, enhanced tumor selectivity and improved disease response [18].

Chemotherapeutic resistance is a trait common to many CSCs and is mediated by diverse cellular processes such enhanced DNA damage repair or rapid drug efflux [19]. Nanoparticles (NPs) can sequester chemotherapeutic agents at a high concentration and release them

${ }^{*}$ Corresponding author: Suzy V. Torti, Department of Molecular, Microbial and Structural Biology, University of Connecticut School of Medicine, 263 Farmington Avenue, Farmington, CT 06030, USA, E-mail: storti@uchc.edu

Received June 01, 2012; Accepted June 25, 2012; Published July 02, 2012

Citation: Burke AR, Singh RN, Carroll DL, Torti FM, Torti SV (2012) Targeting Cancer Stem Cells with Nanoparticle-Enabled Therapies. J Mol Biomarkers Diagn S8:003. doi:10.4172/2155-9929.S8-003

Copyright: ( 2012 Burke AR, et al. This is an open-access article distributed under the terms of the Creative Commons Attribution License, which permits unrestricted use, distribution, and reproduction in any medium, provided the original author and source are credited 
within the cancer cell following uptake by CSCs, potentially overcoming such resistance mechanisms. The addition of targeting ligands to the surface of NPs may increase both target selectivity and internalization. Recently, several groups have explored the application of such NP drug delivery platforms for the selective treatment of CSCs.

In a study by Lim et al., researchers investigated the efficacy of a proprietary polymer-encapsulated curcumin NP formulation (termed NanoCurc ${ }^{\mathrm{TM}}$ ) for the treatment of brain tumor stem cells [20]. The NP formulation greatly increased the bioavailability of curcumin, and following treatment of four distinct brain cancer cell lines with NanoCurc ${ }^{\mathrm{TM}}$ increased rates of cell cycle arrest, apoptosis, and dosedependent decreases in growth and clonogenicity were observed. Critically, this treatment correlated with a $>50 \%$ decrease in the $\mathrm{CD}_{133^{+}}$stem cell population in two of the cell lines tested, suggesting that this therapy may have activity in the CSC fraction of some brain tumors [20].

Recent work by Mamaeva and coauthors describes the use of folate-conjugated mesoporous silica nanoparticles for the in vivo disruption of Notch signaling by the gamma secretase inhibitor DAPT [N-(N-((3,5-Difluorophenacetyl))-L-alanyl)-S-phenylglycerin t-butyl ester] [21]. Notch, like Wnt/ $\beta$-Catenin, Hedgehog and other key developmental signaling pathways, has been implicated in the maintenance of the CSC pool of many tumors [22], and therapies that attenuate these pathways are being investigated for the treatment of several malignancies $[23,24]$. Initial studies characterizing the effects of the nanoparticles in breast cancer cell cultures demonstrated folate receptor mediated uptake (with high-expressing cell lines exhibiting greater uptake relative to low-expressers) along with dose-dependent inhibition of Notch intracellular domain (NICD)cleavage, a standard metric of Notch pathway activation [21]. Encouragingly, peritumoral injection of mesoporous NPs in tumor-bearing mice lead to significant tumor growth suppression, whereas free drug exhibited little effect. Moreover, it was shown that drug potency was maintained with oral dosing of the construct; an important consideration for future clinical translation.

Wang et al. described the use of a novel anti-CD44 antibody conjugated liposome to target an aggressive hepatocellular CSC with enhanced tumorigenicity and metastatic potential that over expressed both CD44 and CD90 [25]. The targeted liposomes were loaded with doxorubicin and then injected intravenously into tumor-bearing mice, resulting in a seven-fold higher drug accumulation in tumors relative to free drug, which corresponded with decreased tumor volume. Encouragingly, this effect was seen in the absence of significant changes in mouse body mass. Treatment with free drug produced similar decreases in tumor burden but with an attendant $>30 \%$ loss of body mass in exposed animals. Alternatively, the authors were able to simultaneously perform tumor imaging and use gene therapy to treat the cancer by using the targeted liposome to deliver a triple fusion plasmid, consisting of gene expression cassettes for red fluorescence protein (RFP), renilla luciferase (Rluc), and a truncated herpes simplex virus thymidine kinase (HSV-TTK) gene. Treatment of tumor-bearing mice with the combination of HSV-TTK liposome and ganciclovir (a cytotoxic thymidine kinase substrate) caused a robust increase in tumor-localized apoptosis with minimal impact on normal tissues. The application of NPs for gene therapy will be discussed in more detail below.

\section{Nanoparticle-enabled nucleic acid delivery vectors targeting CSCs}

Nucleic acid-based therapies (such as RNAi) have long offered the promise of a molecularly-tailored intervention for cancer treatment, through the knockdown of vital oncogenes or disruption of tumoressential signaling networks. Despite the theoretical potential, the clinical introduction of these therapies has been slowed by their unfavorable native pharmacokinetics and poor tumor uptake in vivo. The incorporation of therapeutic nucleic acids into NP delivery vectors is one approach being investigated to overcome these limitations.

Work by Liu et al. describes a method to overcome chemotherapy resistance in colon cancer stem cells through the siRNA-mediated knockdown of the drug efflux protein multidrug resistance 1 (MDR1), which often is over-expressed in CSCs [26]. Utilizing a moderatethroughput approach, the authors generated libraries of lipid nanocarriers composed of varying ratios of cationic polyethylenimine $\left(\mathrm{PEI}_{1200}\right)$, polyethylene glycol (PEG) and a biodegradable lipid crosslinker. Electrostatic complexes formed by mixing siRNA with these particles with a charge ratio of 1:16 were screened for knockdown efficiency, and optimized nanocarrier formulations achieved $>90 \%$ silencing. Treatment of colon cancer stem cells with lipid nanocarriers containing MDR1-directed siRNA led to efficient MDR1 knockdown and sensitized the cells to subsequent paclitaxel treatment [26].

MicroRNAs (miRs) have garnered interest for their ability to coordinately regulate multiple intracellular signaling networks simultaneously [27]. Two recent publications explore the efficacy of NP-delivered, tumor-suppressive miRs for the treatment of head and neck and pancreatic cancers and their constituent stem cells. In one, Piao et al. used a cationic lipid nanoparticle delivery system to express pre-miR 107 in target cells [28]. Mir-107 is a known tumorsuppressive miR capable of regulating key proliferation and survival genes such as protein kinase $\mathrm{C} \varepsilon(\mathrm{PKC} \varepsilon)$, cyclin-dependent kinase 6 (CDK6) and hypoxia-inducible factor 1- $\beta$ (HIF1- $\beta$ ). Treatment of a model of head and neck squamous cell carcinoma (HNSCC) with this NP led to a reduction of cellular clonogenicity, invasion and migration [28]. Moreover, therapeutic expression of pre-miR-107 resulted in a significant down-regulation of stem cell transcription factors Nanog, Oct3/4 and Sox 2 along with diminished tumor sphere forming efficiency in these same cell lines, suggesting an inhibitory effect on resident CSCs. Accordingly, systemic delivery of NP-encapsulated premiR-107 retarded tumor growth and significantly increased survival in HNSCC tumor-bearing mice [28].

In the second study, Pramanik et al. employed a similar cationic liposomal delivery system to investigate the therapeutic utility of forced re-expression of tumor-suppressive miRs 34a and 143/14 for the treatment of pancreatic cancer [29]. Similar to miR-107, these miRs are frequently down regulated during carcinogenesis [30,31]. Intravenous administration of miR-34a or 143/145-complexed liposomes in mice produced increased intratumoral apoptosis and growth delays in pancreatic cancer xenografts and orthotopic tumor models. Furthermore, miR-34a re-expression caused significant downregulation of pancreatic CSC markers aldehyde dehydrogenase 1 (ALDH1) and CD44, suggesting that miR-34a therapy may be effective for the treatment of both stem and non-stem pancreatic tumor cells [32].

\section{Nanoparticle-mediated hyperthermia for CSCs}

Heat-based therapies, which involve elevating specific regions of the body to temperatures in excess of $43^{\circ} \mathrm{C}$ (hyperthermia) or $55^{\circ} \mathrm{C}$ (thermal ablation), are established therapeutic options for the treatment of refractory tumors and metastases. Raising the temperature of a tumor into a supra physiologic range enhances chemotherapeutic 
uptake and tumor oxygenation (a positive modifier of response to radiotherapy), as well as exerting direct cytotoxic effects [33]. While disease responses to hyperthermal therapies have been widely observed, their clinical implementation has been limited due to the nonspecific heating of normal tissues and consequent treatment-limiting toxicities. Recent evidence also suggests that CSCs are resistant to many standard thermal therapies. However, developmental advances in biocompatible near-infrared and radio frequency (RF) energy absorbing nanoparticles offer the possibility of generating tumor-specific thermal therapy in a minimally-invasive manner. In this application, nanoparticles are localized to the target lesion either by direct injection or through intravenous administration followed by either passive or targeting moiety-assisted accumulation at the tumor site. The tumor is then irradiated with either NIR or RF energy to stimulate the nanoparticles and locally generate heat within the tumor, leading to cancer cell death.

This technique was first described by Hirsch et al. using goldcoated, silica core nanoshells [34]. Similar findings have since been reported with the use of graphene [35], single [36] and multiwalled [37,38] carbon nanotubes and gold nanorods [39]. In recent work, Burke and co-authors directly investigated the response of breast cancer stem cells (BCSCs) to both conventional and nanoparticlemediated hyperthermia (NMH) to determine the relative efficacy of each approach for the treatment of these cells [40]. They reported that BCSCs were significantly more resistant to the cytotoxic effects of conventional hyperthermia as compared to non-stem breast cancer cells and that this resistance was mediated, in part, by high basal expression levels of heat shock protein 90 (HSP 90). Treatment of a mixed population of stem and non-stem breast cancer cells with conventional hyperthermia led to a significant enrichment of BCSCs in the surviving fraction of cells. In contrast, the researchers were able to abrogate the resistance to hyperthermia observed in BCSCs following conventional treatment through the use of $\mathrm{NMH}$ [40]. It this study, the researchers were able to generate precise temperature increases in target cells and tissues by exposing the cells to polyethylene glycol coated multiwalled carbon nanotubes that were then heated using a low power, $1064 \mathrm{~nm}$ NIR laser. Treatment of the BCSCs with this form of NMH resulted in robust cell death that was proportional to laser exposure time. $\mathrm{NMH}$ treatments, but not conventional hyperthermia, led to rapid membrane permeabilization and necrotic death in treated cells, and were equivalently effective at treating both cancer stem cells and non-stem cancer cells. Encouragingly, use of NMH in mice bearing BCSC-driven tumors lead to complete tumor regression and $100 \%$ survival, whereas control groups exhibited $>80 \%$ mortality at identical time points. Based on these findings, NMH may represent a rapid, minimally invasive approach for the simultaneous elimination of stem and non-stem cellular components of tumors [40].

$\mathrm{NMH}$ can also sensitize CSCs to other treatments such as ionizing radiation exposure. This type of bipartite therapeutic approach was investigated by Atkinson et al. [41]. The authors used gold nanoshells in combination with NIR laser irradiation to generate mild $\left(\approx 42^{\circ} \mathrm{C}\right)$ hyperthermia in target cells and tumors and investigated the combined effects of focal hyperthermia and ionizing radiation treatment. Using two independent animal models of breast cancer they confirmed that the stem cell fraction of the tumors (identified by $\mathrm{CD} 29^{+} / \mathrm{CD} 24^{+} /$ Lin antigen profiles or ALDH1 enzymatic activity) was resistant to radiation monotherapy and became enriched in the population of tumor cells surviving treatment, as had been previously shown [42]. They went on to show that this effect could be prevented by the addition of hyperthermia immediately following radiotherapy, which led to a $>50 \%$ reduction in the size of the CSC fraction. Moreover, cells from tumors treated with the combined therapy displayed reduced tumorigenicity and gave rise to less aggressive, more differentiated tumors (when formed) following transplantation into new hosts. These results suggested that the combination therapy durably altered the native behavior of the CSC fraction and may represent a promising approach for the treatment of CSC-harboring breast tumors. Human clinical trials using these particles under the trade name Aurolase ${ }^{\circledR}$ are currently underway.

Finally, a recent report describes a novel extension of the $\mathrm{NMH}$ technique to target invasive CSCs in systemic blood circulation. In a proof-of-principle study, Galanzha et al. demonstrated the use of photoacoustic (PA)/photothermal (PT) in vivo flow cytometry for the detection and elimination of circulating cancer stem cells [43]. The authors conjugated NIR-absorbing gold plated single-walled carbon nanotubes (GNTs) and spherical magnetic nanoparticles (MNPs) to folate or anti-human CD44 antibodies, and used these particles to selectively label circulating human breast cancer stem cells (which over express CD44 [6]) with nanoparticles (NPs). Cells with bound nanoparticles could then be specifically identified by detection of photoacoustic waves generated by the nanoparticle-labeled cells following excitation using a low powered laser [44,45]. Using this method, the authors demonstrated that rare $\mathrm{CD} 44^{+}$circulating cancer stem cells could be detected in the vasculature of nude mice which bore human breast cancer xenografts. The authors suggested that these cells could be ablated by photothermal effect following extended irradiance with NIR. As circulating CSCs are thought to be the primary drivers of metastatic spread, this technology offers a method by which these cells may be purged from the vasculature of cancer patients to reduce the incidence of metastatic disease.

\section{Conclusions}

Cancer stem cells offer an attractive target for therapeutic intervention because therapies that ablate this critical tumor constituency offer the promise of durable disease remission and longterm survival of cancer patients. This review discussed three promising nanomedical approaches for the selective treatment of both tumors and their resident CSC populations. Each approach leverages the emergent properties of distinct nano-scale material formulations to enhance both tumor and CSC-specific drug accumulation and therapeutic effect.

It is encouraging to note that despite a challenging regulatory environment, several nanomedical technologies (including the Aurolase $^{\circledR}$ nanoshell technology discussed above) are already undergoing clinical trials. While future advancements will be necessary to safely transition the investigational nanoparticles detailed in this review into the clinic, the lessons learned by pioneering treatments like Aurolase $^{\circledR}$ will inform the rational design and development of future nanomedicines for targeted cancer therapy.

\section{Acknowledgements}

This work was supported in part by grants R01CA12842 from the National Institutes of Health (SVT), Department of Defense Breast Cancer Research Program Predoctoral Traineeship Award W81XWH-10-1-0332 (ARB) and by a Grant-In-Aid of Research from the National Academy of Sciences, administered by Sigma Xi, the Scientific Research Society G20100315151746 (ARB). R.S. was supported in part by training grant T32CA079448 and by National Institutes of Health grant K99CA154006.

\section{References}

1. Charafe-Jauffret E, Monville F, Ginestier C, Dontu G, Birnbaum D, et al (2008) Cancer Stem Cells in Breast: Current Opinion and Future Challenges. Pathobiology 75: 75-84. 
2. Ponti D, Costa A, Zaffaroni N, Pratesi G, Petrangolini G, et al. (2005) Isolation and in vitro propagation of tumorigenic breast cancer cells with stem/progenitor cell properties. Cancer Res 65: 5506-5511.

3. Prince ME, Sivanandan R, Kaczorowski A, Wolf GT, Kaplan MJ, et al. (2007) Identification of a subpopulation of cells with cancer stem cell properties in head and neck squamous cell carcinoma. Proc Natl Acad Sci USA 104: 973-978.

4. Clarke MF, Dick JE, Dirks PB, Eaves CJ, Jamieson CH, et al. (2006) Cancer stem cells-perspectives on current status and future directions: AACR workshop on cancer stem cells. Cancer Res 66: 9339-9344.

5. Bonnet D, Dick JE (1997) Human acute myeloid leukemia is organized as a hierarchy that originates from a primitive hematopoietic cell. Nat Med 3: 730737.

6. Al-Hajj M, Wicha MS, Benito-Hernandez A, Morrison SJ, Clarke MF (2003) Prospective identification of tumorigenic breast cancer cells. Proc Natl Acad Sci USA 100: 3983-3988

7. Kondo T, Setoguchi T, Taga T (2004) Persistence of a small subpopulation of cancer stem-like cells in the C6 glioma cell line. Proc Natl Acad Sci USA 101: $781-786$

8. Eramo A, Lotti F, Sette G, Pilozzi E, Biffoni M, et al. (2008) Identification and expansion of the tumorigenic lung cancer stem cell population. Cell Death Differ 15: 504-514.

9. Li C, Heidt DG, Dalerba P, Burant CF, Zhang L, et al. (2007) Identification of pancreatic cancer stem cells. Cancer Res 67: 1030-1037.

10. Lee CJ, Dosch J, Simeone DM (2008) Pancreatic cancer stem cells. J Clin Oncol 26: 2806-2812.

11. Boman BM, Huang E (2008) Human colon cancer stem cells: a new paradigm in gastrointestinal oncology. J Clin Oncol 26: 2828-2838.

12. Cocciadiferro L, Miceli V, Kang KS, Polito LM, Trosko JE, Carruba G (2009) Profiling cancer stem cells in androgen-responsive and refractory human prostate tumor cell lines. Ann NY Acad Sci 1155: 257-262.

13. Schatton T, Murphy GF, Frank NY, Yamaura K, Waaga-Gasser AM, et al (2008) Identification of cells initiating human melanomas. Nature 451: $345-349$.

14. Bussolati B, Bruno S, Grange C, Ferrando U, Camussi G (2008) Identification of a tumor-initiating stem cell population in human renal carcinomas. Faseb $\mathrm{J}$ 22: $3696-3705$

15. Dean M, Fojo T, Bates S (2005) Tumour stem cells and drug resistance. Nat Rev Cancer 5: 275-284.

16. Li X, Lewis MT, Huang J, Gutierrez C, Osborne CK, et al. (2008) Intrinsic resistance of tumorigenic breast cancer cells to chemotherapy. J Natl Cancer Inst 100: 672-679.

17. Shapira A, Livney YD, Broxterman HJ, Assaraf YG (2011) Nanomedicine for targeted cancer therapy: towards the overcoming of drug resistance. Drug Resist Update 14: 150-163.

18. Ahmed N, Fessi H, Elaissari A (2012) Theranostic applications of nanoparticles in cancer. Drug Discov Today 17: 928-34.

19. Vinogradov S, Wei $X$ (2012) Cancer stem cells and drug resistance: the potential of nanomedicine. Nanomedicine UK 7: 597-615.

20. Lim KJ, Bisht S, Bar EE, Maitra A, Eberhart CG (2011) A polymeric nanoparticle formulation of curcumin inhibits growth, clonogenicity and stem-like fraction in malignant brain tumors. Cancer Biol Ther 11: 464-473.

21. Mamaeva V, Rosenholm JM, Bate-Eya LT, Bergman L, Peuhu E, et al. (2011) Mesoporous silica nanoparticles as drug delivery systems for targeted inhibition of Notch signaling in cancer. Mol Ther 19: 1538-1546.

22. Zhou BBS, Zhang H, Damelin M, Geles KG, Grindley JC, et al. (2009) Tumourinitiating cells: challenges and opportunities for anticancer drug discovery. Nature Rev Drug Discov 8: 806-823.

23. McDermott SP, Wicha MS (2010) Targeting breast cancer stem cells. Mol Oncol 4: 404-419.

24. Wu X, Chen H, Wang X (2012) Can lung cancer stem cells be targeted for therapies? Cancer Treat Rev 38:580-588.

25. Yang ZF, Ho DW, Ng MN, Lau CK, Yu WC, et al. (2008) Significance of CD90+cancer stem cells in human liver cancer. Cancer Cell 13: 153-166.
26. Liu C, Zhao G, Liu J, Ma N, Chivukula P et al. (2009) Novel biodegradable lipid nano complex for siRNA delivery significantly improving the chemosensitivity of human colon cancer stem cells to paclitaxel. J Control Release 140: 277-283.

27. Bader AG, Brown D, Stoudemire J, Lammers $P$ (2011) Developing therapeutic microRNAs for cancer. Gene Ther 18:1121-1126.

28. Piao L, Zhang M, Datta J, Xie X, Su T, et al. (2012) Lipid-based Nanoparticle Delivery of pre-miR-107 inhibits the tumorigenicity of head and neck squamous cell carcinoma. Mol Ther 20: 1261-1269.

29. Pramanik D, Campbell NR, Karikari C, Chivukula R, Kent OA, et al. (2011) Restitution of tumor suppressor microRNAs using a systemic nanovector inhibits pancreatic cancer growth in mice. Mol Cancer Ther 10: 1470-1480.

30. Yin D, Ogawa S, Kawamata N, Leiter A, Ham M, et al. (2012) miR-34a functions as a tumor suppressor modulating EGFR in glioblastoma multiforme. Oncogene.

31. Ugras S, Brill E, Jacobsen A, Hafner M, Socci ND, et al. (2011) Small RNA sequencing and functional characterization reveals MicroRNA-143 tumor suppressor activity in liposarcoma. Cancer Res 71: 5659-5669.

32. Pramanik D, Campbell NR, Karikari C, Chivukula R, Kent OA, et al. (2011) Restitution of tumor suppressor microRNAs using a systemic nanovector inhibits pancreatic cancer growth in mice. Mol Cancer Ther 10: 1470-1480.

33. Issels RD (2008) Hyperthermia adds to chemotherapy. Eur J Cancer 44 2546:2554.

34. Hirsch LR, Stafford RJ, Bankson JA, Sershen SR, Rivera B, et al. (2003) Nanoshell-mediated near-infrared thermal therapy of tumors under magnetic resonance guidance. Proc Natl Acad Sci USA 100: 13549-13554

35. Yang K, Zhang S, Zhang G, Sun X, Lee ST, et al. (2010) Graphene in mice: ultrahigh in vivo tumor uptake and efficient photothermal therapy. Nano Lett 10: $3318-3323$

36. Kam NW, O'Connell M, Wisdom JA, Dai H (2005) Carbon nanotubes as multifunctional biological transporters and near-infrared agents for selective cancer cell destruction. Proc Natl Acad Sci U S A 102: 11600-11605.

37. Burke A, Ding X, Singh R, Kraft RA, Levi-Polyachenko N, et al. (2009) Longterm survival following a single treatment of kidney tumors with multiwalled carbon nanotubes and near-infrared radiation. Proc Natl Acad Sci USA 106 12897-12902.

38. Ding X, Singh R, Burke A, Hatcher H, Olson J, et al. (2011) Development of iron-containing multiwalled carbon nanotubes for MR-guided laser-induced thermotherapy. Nanomedicine UK 6: 1341-1352.

39. Huang X, El-Sayed IH, Qian W, El-Sayed MA (2006) Cancer cell imaging and photothermal therapy in the near-infrared region by using gold nanorods. J Am Chem Soc 128: 2115-2120.

40. Burke AR, Singh RN, Carroll DL, Wood JC, D'Agostino RB, et al. (2012) The resistance of breast cancer stem cells to conventional hyperthermia and their sensitivity to nanoparticle-mediated photothermal therapy. Biomaterials 33 2961-2970.

41. Atkinson RL, Zhang M, Diagaradjane $P$, Peddibhotla S, Contreras $A$, et al (2010) Thermal enhancement with optically activated gold nanoshells sensitizes breast cancer stem cells to radiation therapy. Sci Transl Med 2: 55-79.

42. Phillips TM, McBride WH, Pajonk F (2006) The response of CD24(-/low)/CD44+ breast cancer-initiating cells to radiation. J Natl Cancer Inst 98: 1777-1785.

43. Galanzha El, Kim JW, Zharov VP (2009) Nanotechnology-based molecular photoacoustic and photothermal flow cytometry platform for in-vivo detection and killing of circulating cancer stem cells. J Biophotonics 2: 725-735.

44. Galanzha El, Shashkov EV, Kelly T, Kim JW, Yang L, et al. (2009) In vivo magnetic enrichment and multiplex photoacoustic detection of circulating tumour cells. Nature Nanotechnol 4:855-860.

45. Kim JW, Galanzha El, Shashkov EV, Moon HM, Zharov VP (2009) Golden carbon nanotubes as multimodal photoacoustic and photothermal high-contrast molecular agents. Nature Nanotechnol 4: 688-694.

This article was originally published in a special issue, Potential Biomarkers and Therapeutic Targets in Cancer Stem Cells handled by Editor(s). Dr. Murielle Mimeault, University of Nebraska Medical Center, USA 